AnaleS Cientificos
ISSN 2519-7398 (Versión electrónica)

ARTÍCULO ORIGINAL - RESEARCH ARTICLE http://dx.doi.org/10.21704/ac.v81i2.1679

\title{
RESPUESTA DEL INICIO DEL SECADO PARCIAL DE RAÍCES EN EL RENDIMIENTO DEL CULTIVO DE CAMOTE (Ipomoea batatas)
}

\section{Response of the beginning of the partial root-zone drying irrigation in the performance of the sweet potato culture (Ipomoea batatas)}

\author{
Miguel A. Sánchez D. ${ }^{1 * D}$; Jesús A. Mejía M. ${ }^{1}$; ; Duaner A. Juli T. ${ }^{1}$; Gilberto Rodríguez S. ${ }^{1}$; \\ José B. Arapa Q. ${ }^{1}$ \\ ${ }^{1}$ Facultad de Ingeniería Agrícola Universidad Nacional Agraria La Molina, 15024, Lima, Perú. \\ *E-mail: msanchez@lamolina.edu.pe
}

Recibido: 20/06/2019; Aceptado: 15/12/2020; Publicado: 30/12/2020

\begin{abstract}
The objective of this study was evaluate, the response of the beginning of the Partial Root-Zone Drying Irrigation (PRD) in the performance of the sweet potato culture, for both the Huambachero and Paramonguino varieties. For this investigation drip irrigation was applied in two ways; with $100 \%$ of irrigation sheet and PRD with $50 \%$ of irrigation sheet, with three different beginnings of PRD; at 23, 33 and 45 days after sowing, watered with a frequency of 2 days. The yields for the control treatment were 42,1 tha ${ }^{-1}$ and 39,2 tha ${ }^{-1}$ for both the Huambachero and Paramonguino varieties and for the early PRD of 23, 33 and 45 days of 37,4 t.ha ${ }^{-1}, 32,7$ t.ha $^{-1}$ and 35,1 tha ${ }^{-1}$, in the Huambachero variety and 30,0 t.ha- ${ }^{-1}, 31,4$ t.ha $^{-1}$ and 32,6 t.ha ${ }^{-1}$ in the Paramonguino variety.The water application for both varieties was: $5368,9 \mathrm{~m}^{3} \cdot \mathrm{ha}^{-1}$ in the control treatment, $3185,54 \mathrm{~m}^{3} \cdot \mathrm{ha}^{-1}, 3429,47 \mathrm{~m}^{3} \cdot \mathrm{ha}^{-1}$ and $3713,26 \mathrm{~m}^{3}$.ha $\mathrm{a}^{-1}$ for the PRD of 23, 33 and 45 days. Water productivity in the Huambachero variety were: control treatment 7,85 kg.m $\mathrm{m}^{-3}$, and for the beginning of PRD of 23,33 and 45 days of $10,91 \mathrm{~kg} \cdot \mathrm{m}^{-3}, 9,52 \mathrm{~kg} \cdot \mathrm{m}^{-3}$ and 9,46 $\mathrm{kg} \cdot \mathrm{m}^{-3}$ respectively. In the Paramonguino variety they were: control treatment $7,30 \mathrm{~kg} \cdot \mathrm{m}^{-3}$ and for the early days of PRD of 23, 33 and 45 days of $9,42 \mathrm{~kg} \cdot \mathrm{m}^{-3}, 9,15 \mathrm{~kg} \cdot \mathrm{m}^{-3}$ and $8,78 \mathrm{~kg} \cdot \mathrm{m}^{-3}$ respectively.
\end{abstract}

Keywords: irrigation; drip; Partial Root-Zone Drying Irrigation; yield; sweet potato; water productivity.

\section{RESUMEN}

El objetivo del presente trabajo es evaluar, la respuesta del inicio del Secado Parcial de Raíces (SPR) en el rendimiento del cultivo de camote, para las variedades Huambachero y Paramonguino. Se utilizó riego por goteo; con láminas de riego del 100\% de la evapotranspiración del cultivo (tratamiento control) y el 50\% de la evapotranspiración del cultivo correspondiente al SPR, con tres inicios diferentes del SPR; a los 23, 33 y 45 días después de la siembra, regado con una frecuencia de 2 días. Los rendimientos para el tratamiento control fueron de 42,1 tha ${ }^{-1}$ y 39,2 t.ha ${ }^{-1}$ tanto para las variedades Huambachero y Paramonguino, para los inicios de SPR de 23, 33 y 45 días de 37,4 t.ha ${ }^{-1}, 32,7$ t.ha $^{-1}$ y 35,1 t.ha ${ }^{-1}$, en la variedad Huambachero y de 30,0 t.ha ${ }^{-1}, 31,4$ t.ha ${ }^{-1}$ y 32,6 t.ha ${ }^{1}$ en la variedad Paramonguino. La eficiencia de uso de agua en la variedad Huambachero para el tratamiento control fue 7,85 kg.m $\mathrm{m}^{-3}$ y para los inicios de SPR de 23, 33 y 45 días de $10,91 \mathrm{~kg} \cdot \mathrm{m}^{-3}, 9,52 \mathrm{~kg} \cdot \mathrm{m}^{-3}$ y $9,46 \mathrm{~kg} \mathrm{~m}^{-3}$ respectivamente. En la variedad Paramonguino para el tratamiento control fue de 7,30 kg.m ${ }^{-3}$ y para los inicios de 
SPR de los 23, 33 y 45 días de 9,42 kg.m $\mathrm{m}^{-3}, 9,15 \mathrm{~kg} \cdot \mathrm{m}^{-3}$ y $8,78 \mathrm{~kg} \cdot \mathrm{m}^{-3}$ respectivamente. Se concluye que, la conveniencia del uso del SPR siendo el momento más adecuado del inicio del SPR a los 23 días después de la siembra.

Palabras clave: Goteo; riego parcial; rendimiento; camote; eficiencia de uso de agua; Secado Parcial de Raíces.

\section{Forma de citar el artículo (Formato APA):}

Sánchez. M., Mejía, A., Juli, D., Rodríguez, G., \& Arapa, J. (2020). Respuesta del inicio del secado parcial de raíces en el rendimiento del cultivo de camote (Ipomoea batatas). Anales Científicos. 81(2), 385-394. http://dx.doi.org/10.21704/ac.v81i2.1679

Autor de correspondencia (*): Miguel A. Sánchez D. Email: msanchez@lamolina.edu.pe

(c) Los autores. Publicado por la Universidad Nacional Agraria La Molina.

This is an open access article under the CC BY

\section{INTRODUCCIÓN}

La escasez de agua en la costa peruana, se ha convertido en una de las principales preocupaciones en el ámbito de la agricultura, por lo que se ha visto conveniente tecnificar el riego, para que el uso de este recurso pueda ser óptimo y mejorar las actuales eficiencias del riego por gravedad $40 \%$, riego por aspersión $70 \%$ y riego por goteo $90 \%$, de estas tres el riego por goteo es la más eficiente, siendo además una de sus ventajas el manejo integral de malezas para incrementar el rendimiento y calidad del producto.

El camote (Ipomoea batatas) a menudo se cultiva en tierras comunales con suelos marginales y agua de riego limitada (King et al., 2008), en el futuro, será necesario mejorar la eficiencia del uso del agua en las tierras de secano y de regadío para compensar las necesidades adicionales de riego y satisfacer la demanda de producción de alimento (Doorenbos y Kassam, 1979).

El principal motivo para el uso del sistema de riego por goteo es el ahorro de agua, porque se puede reducir el escurrimiento, evaporación o percolación, no permitiendo que el agua tenga contacto con el follaje o los tallos, evitando enfermedades, así también aumentar el rendimiento, cubriendo las necesidades hídricas de la planta (Shock y Welch, 2013).

Sánchez y Torrecillas (1995), indican que al reducir los aportes hídricos a la planta en periodos fenológicos adecuados para que no afecte sensiblemente a la producción y calidad de la cosecha y cubrir en su plenitud durante el resto del ciclo del cultivo, puede definir la idoneidad del uso del riego deficitario en dicho cultivo, además el déficit de agua en el suelo parece provocar en las raíces la emisión de señales químicas que inducen a su vez en las hojas un efecto de mayor duración en el cierre estomático para prevenir la deshidratación foliar.

Kang y Zhang (2004), dicen que al regar parcialmente un cultivo los lados mojados y secos del sistema radicular se alternan en una frecuencia según los cultivos. Esta técnica tiene el potencial para reducir el uso del agua de cultivos y árboles frutales significativamente, aumentar el vigor del dosel y mantener los rendimientos, en comparación con los métodos convencionales de riego.

Según Molden (1977), la eficiencia del uso del agua generalmente se conoce como la producción total de materia seca por unidad de transpiración $\mathrm{o}$ evapotranspiración (en $\mathrm{m}^{3} \mathrm{o} \mathrm{mm}$ de agua) por el cultivo durante la temporada de crecimiento; y es importante en todas las áreas de producción de plantas. Según Atwell et al. (1999), la eficiencia en el uso del agua es el resultado de toda la colección de plantas y procesos ambientales que funcionan durante la vida de un cultivo para determinar tanto el rendimiento como la evapotranspiración. Esto incluye la capacidad del suelo para capturar y almacenar agua; la capacidad del cultivo para acceder al agua almacenada en el suelo, convertir el agua en biomasa; y convertir la biomasa en índice de cosecha económica. De Pascale et al. (2011), mencionan que los criterios para mejorar la eficiencia del uso del agua pueden implicar el control de procesos fisiológicos que afectan la transpiración y el rendimiento de la planta. El riego deficitario es en algunos casos beneficioso en la mejora de la eficiencia 
del uso del agua, especialmente si coincide con las etapas menos sensibles del crecimiento de la planta (Dalla y Giovanardi, 2000).

El suministro limitado de agua para cumplir con los requisitos de evapotranspiración es una herramienta muy importante para aumentar la eficiencia en el uso del agua. El riego deficitario implica, un aumento gradual en el estrés hídrico de los cultivos, a través de la reducción uniforme de la cantidad de agua aplicada en un determinado momento (Fereres y Soriano, 2007).

Masango (2014), menciona que, para mejorar la eficiencia del uso del agua, se requiere un momento correcto de la aplicación de riego según los requerimientos de agua del cultivo. Esto se puede lograr estimando la humedad del suelo a través de la observación visual del suelo; aplicación de dispositivos/instrumentos relevantes; por ejemplo, tensiómetros, sensores de humedad del suelo y sondas de neutrones; medir el estrés hídrico de la planta y; evaluando y midiendo parámetros como la evapotranspiración y el uso de modelos matemáticos. La adopción de tales métodos para controlar los niveles de estrés hídrico de las plantas puede reducir significativamente las cantidades de aplicación de agua (De Pascale et al., 2011). Asimismo, se han desarrollado modelos informáticos para el monitoreo constante de los parámetros específicos del cultivo y la programación del riego.

Masango (2014), concluye que la eficiencia del uso del agua se puede mejorar mediante la selección de cultivares específicos, el tiempo de cultivo y el entorno específico para el crecimiento de las plantas.

El objetivo del presente trabajo de investigación, se centró en la aplicación del riego parcial deficitario, usando el sistema de riego por goteo, para determinar el momento más adecuado de inicio del secado parcial de raíces (SPR), en el cultivo de dos variedades de camote, en relación a su rendimiento y la eficiencia del uso del agua.

\section{MATERIALES Y MÉTODOS}

La investigación se realizó en la parcela experimental del Departamento de Recursos Hídricos (DRH),
Facultad de Ingeniería Agrícola (FIA), Universidad Nacional Agraria La Molina (UNALM), ubicada en las coordenadas UTM WGS 84 E 287603,46 y N 8663772,82, a una altitud de 235 msnm. La investigación se realizó de octubre del año 2016 a marzo del año 2017.

El experimento se realizó en 20 unidades experimentales de 3 x $6 \mathrm{~m}$ cada uno, con espaciamiento entre laterales de riego de 1,0 $\mathrm{m}$ y espaciamiento entre goteros de $0,3 \mathrm{~m}$ con un caudal de $3,61 \mathrm{~h}^{-1}$, se contó con un reservorio de $500 \mathrm{~m}^{3}$ con cabezal de riego. El material vegetal, con adaptabilidad climática, fueron dos variedades de camote Huambachero y Paramonguino cuyos esquejes provinieron de la localidad de Barranca, respetando las exigencias fitosanitarias libre de patógenos y enfermedades. Las variables medidas fueron el rendimiento y consumo de agua del cultivo y para el análisis estadístico se utilizó el análisis de varianza y la prueba de comparación múltiple de medias de Tukey.

\section{Características físicas y químicas del suelo}

El análisis, de una muestra representativa, fue realizado por el Laboratorio de Análisis de Agua, Suelo, Medio Ambiente y Fertirriego de la FIA de la UNALM (Tabla 1).

Tabla 1. Características físicas y químicas del suelo.

\begin{tabular}{|c|c|c|}
\hline Análisis Mecánico & $\begin{array}{c}\mathrm{CE}(\mathrm{dS} / \mathrm{m}) \\
\text { Relación } \\
1: 1\end{array}$ & $\begin{array}{c}\text { PH } \\
\text { Relación } \\
1: 1\end{array}$ \\
\hline Textura Arena Limo Arcilla & & \\
\hline $\begin{array}{llll}\text { Franco } & & & \\
\text { Arenoso } & 57,84 & 27,28 & 14,88\end{array}$ & 0,77 & 7,59 \\
\hline
\end{tabular}

\section{Características químicas del agua de riego}

El agua para el riego proveniente del río Rímac, es conducido hasta la UNALM por medio de canales revestidos y se deriva hasta el reservorio de $500 \mathrm{~m}^{3}$, para luego ser conducido al área experimental por medio de una bomba de 5 HP de potencia, las características químicas del agua de riego se presentan en la Tabla 2. 
Tabla 2. Características químicas del agua de riego.

\begin{tabular}{cc}
\hline Características & Valor \\
\hline $\mathrm{CEdS} / \mathrm{m}$ & 0,66 \\
$\mathrm{pH}$ & 7,52 \\
Clasificación & $\mathrm{C} 2-\mathrm{S} 1$ \\
Boro ppm & 0,57 \\
\hline
\end{tabular}

\section{Diseño experimental}

Se usó el Diseño Estadístico Completamente al Azar (DCA) con 4 tratamientos y 5 repeticiones, los tratamientos fueron: tratamiento control, tratamiento secado parcial de raíces con inicio del SPR a los 23 días después de la siembra, tratamiento secado parcial de raíces con inicio del SPR a los 33 días después de la siembra y tratamiento riego parcial de raíces con inicio del SPR a los 45 días después de la siembra. Se eligió el inicio de tratamiento de SPR de 23 días después de la siembra porque el cultivo quedo enraizado completamente a los 21 días después de la siembra. El tratamiento control tuvo una lámina de riego con el $100 \%$ de reposición de la evapotranspiración del cultivo, los tratamientos del secado parcial de raíces SPR tuvieron una lámina de riego con una reposición del $50 \%$ de la evapotranspiración del cultivo respectivamente. La elección de esta lámina fue por la respuesta al estrés hídrico ante un agotamiento de la humedad disponible del $50 \%$. El diseño experimental consideró 20 unidades experimentales, 10 con riego parcial y 10 con riego convencional (tratamiento control o testigo), cada unidad experimental tuvo tres laterales de riego separadas por un 1,0 metro entre laterales, el distanciamiento de los goteros para las parcelas con riego convencional fue de $0,3 \mathrm{~m}$, en cambio para las parcelas con riego parcial se tuvo que juntar paralelamente dos tuberías de riego, los que tuvieron goteros separados a $0,6 \mathrm{~m}$ tal que al juntar desfasadamente las tuberías se tuvo un lateral con goteros distanciados a 0,3 m. En la Figura 1, se puede observar la disposición de las 10 unidades con riego convencional y 10 unidades con riego deficitario modalidad secado parcial de raíces (SPR).

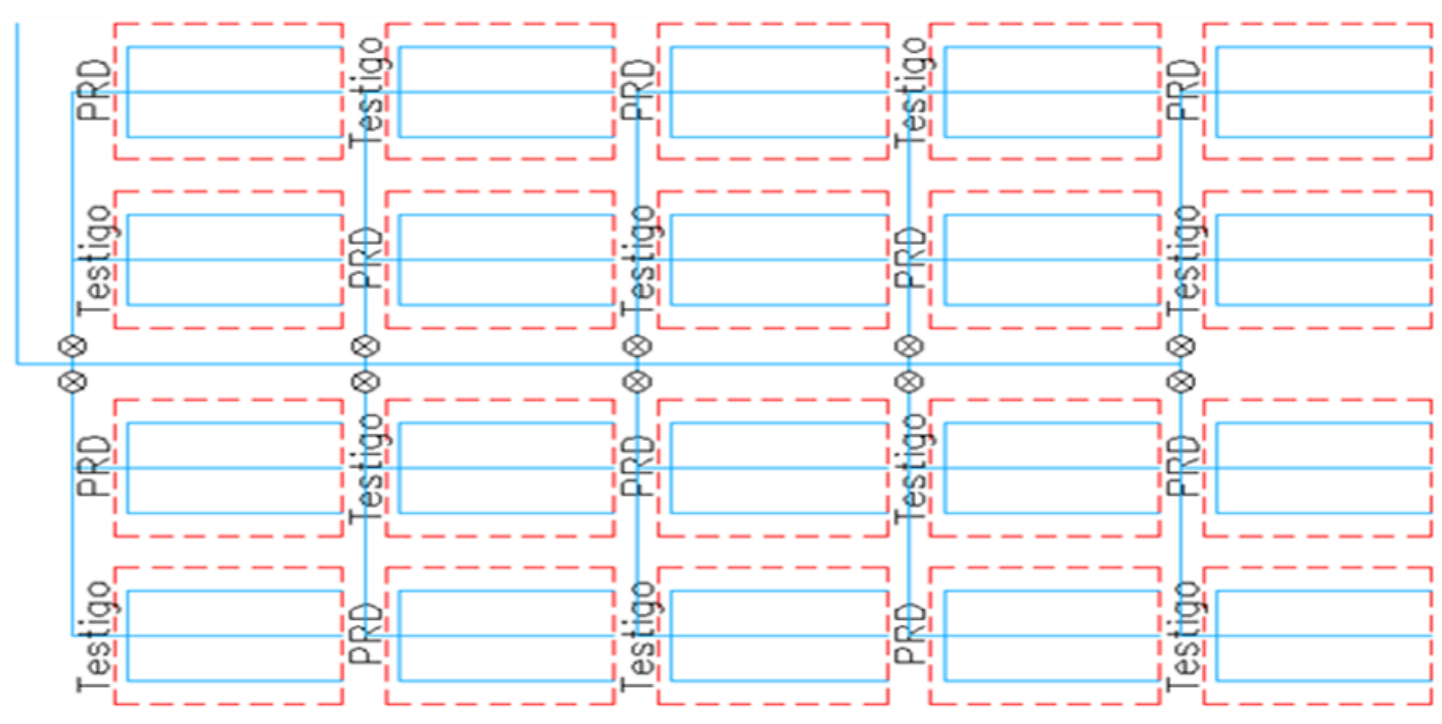

Figura 1. Disposición de unidades experimentales.

En las Figuras 2 y 3, se observa la disposición y comportamiento de los goteros en cada riego, todo ello al operar alternadamente las válvulas ramal A y B de las tuberías de riego. El círculo achurado representa la parte regada que humedece aproximadamente el $50 \%$ de la zona de raíz de la planta de camote.
Los goteros en cada tubería de riego están separados por $60 \mathrm{~cm}$ y las plantas tienen un distanciamiento de siembra $30 \mathrm{~cm}$. 


\section{Riego de las parcelas}

Para el riego se consideró la evapotranspiración medida del cultivo en milímetros obtenido del tanque de evaporación clase A, ubicada en la parcela experimental del área de estudio, fue necesario usar el caudal del gotero y el área que ocupaba cada planta, para que por medio de una relación matemática se pudo obtener el tiempo de riego en las parcelas con cultivo de camote para ambas variedades. La relación matemática es la siguiente:

$T r=\frac{\operatorname{Le} x A \times 60}{q}$

Donde:
Tr: $\quad$ Tiempo de riego (min)

Le: Lamina evaporada ( $\mathrm{mm}$ )

A: $\quad$ Área de plantas $\left(\mathrm{m}^{2}\right)$

q: $\quad$ Caudal de gotero $\left(1^{*} \mathrm{~h}^{-1}\right)$

El cultivo fue sembrado en un área de $566,68 \mathrm{~m}^{2}$ con una pendiente de $4,86 \mathrm{~cm}$ de desnivel vertical en $6 \mathrm{~m}$ de longitud horizontal en el surco $(0,81 \%)$, tres hileras por cada parcela separadas por $1,0 \mathrm{~m}$ y los esquejes sembrados a cada $30 \mathrm{~cm}$ haciendo así 15 esquejes por surco con un total de 45 esquejes por unidad experimental, el periodo vegetativo fue de 150 días, tiempo en el cual se pudo determinar el nivel de crecimiento, desarrollo del cultivo de camote, así como las actividades programadas para el experimento.

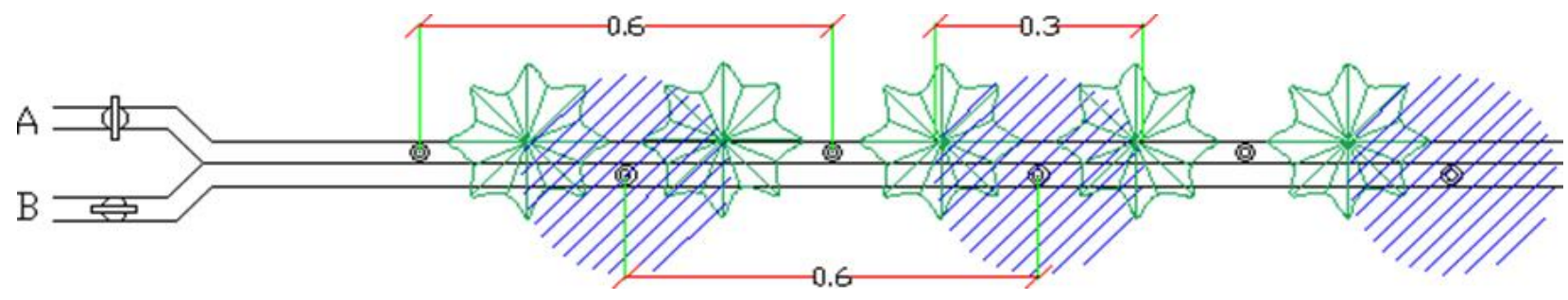

Figura 2. Apertura de la válvula A de la manguera

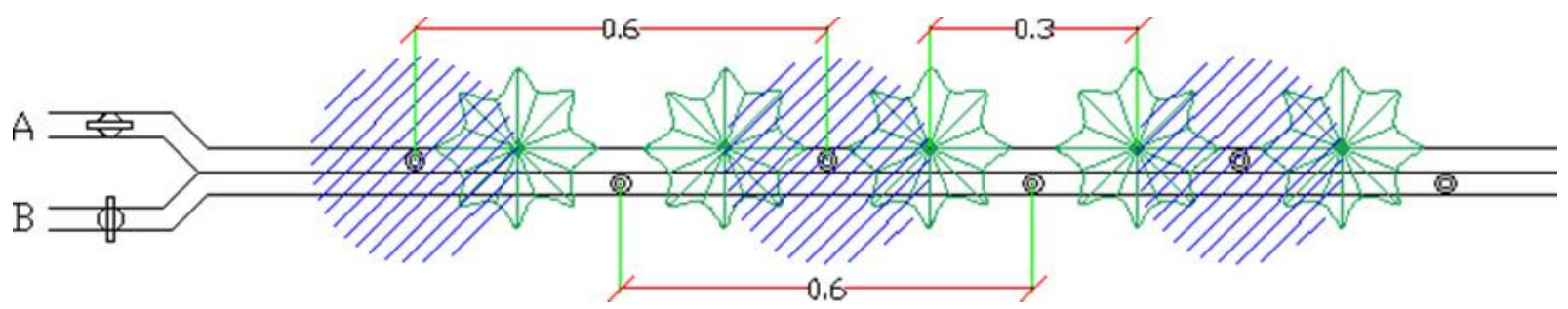

Figura 3. Apertura de la válvula B de la manguera.

\section{RESULTADOS Y DISCUSIÓN}

\section{Rendimiento}

Masango (2014), menciona que la raíz reservante del camote en peso fresco, se considera el rendimiento económico del cultivo. En la literatura, numerosos estudios muestran que la frecuencia de aplicación de agua $y / o$ riego puede reducir o aumentar significativamente el rendimiento de la raíz reservante (Ravi et al., 2009). La producción total acumulada de la raíz reservante en fresco para ambas variedades de camote considerando que el área de cada planta es de $0,24 \mathrm{~m}^{2}$ los rendimientos en toneladas por hectárea (Tabla 3 ).

El rendimiento del tratamiento Control o Testigo de la variedad Huambachero, fue de 42,1 tha ${ }^{-1}$, y de la variedad Paramonguino de 39,18 tha ${ }^{-1}$, los cuales representan el $100 \%$ de la producción, para que los demás tratamientos puedan ser comparados. Para ambas variedades el tratamiento SPR de inicio de riego parcial a los 23 y 45 días después de la siembra, muestran una disminución del rendimiento en un $17 \%$. Önder et al. (2015), reportaron que un 
déficit de agua a un nivel del 20 al $40 \%$ bajo el método de riego por goteo provocó una disminución del rendimiento del cultivo del 25 al $50 \%$. Nedunchezhiyan et al. (2012), reportaron un rendimiento promedio de raíz reservante del camote que varía entre 20 y 25 t.ha ${ }^{-1}$, mientras que Mbwaga et al. (2007), encontraron que 27,3 t.ha ${ }^{-1}$ fue el rendimiento de raíz reservante más alto en las diferentes zonas agroecológicas de Tanzania. Somasundaram y Mithra (2008), informaron un rendimiento promedio de raíz de 31,05 t.ha $^{-1}$ en un experimento para determinar la variación en el rendimiento a diferentes niveles de agua del suelo.

En Mozambique et al. (2001), realizaron un experimento para determinar el efecto de la disponibilidad de agua sobre el rendimiento del camote y se logró un rendimiento de raíz reservante de 34,2 t.ha-1 en la cosecha final. En Sudáfrica, Laurie et al. (2012) reportaron un rendimiento de raíz reservante total que varía entre 7,5 y 48,9 t.ha${ }^{1}$.En Perú, Sánchez y Rivera (2018), en un experimento en condiciones de costa, obtuvieron rendimientos de 62,75 t.ha $^{-1}$ para el riego por goteo intermitente y 57,16 t.ha $^{-1}$ para el riego por goteo convencional en el cultivo de camote INIA 320.

\section{Análisis estadístico del rendimiento}

El análisis de varianza (ANVA) para la variable rendimiento nos indica que no existe diferencias significativas entre tratamientos tanto para la variedad Huambachero como para la variedad Paramonguino, asimismo la prueba de comparación múltiple de medias de Tukey nos indica que no hay diferencias significativas entre el testigo y tratamiento del secado parcial de raíces SPR, en las Tablas 4 y 5 se muestra lo indicado.

En la Figura 4, se puede apreciar que, en todos los tratamientos, se obtuvo un rendimiento mayor en la variedad Huambachero. El comportamiento del rendimiento en la variedad Huambachero, no sigue una tendencia como lo hace la variedad Paramonguino, el cual sigue una forma escalonada, pero de acuerdo a las pruebas estadísticas en ambas variedades no existe significación, al comparar los tratamientos con riego parcial deficitario.

Tabla 3. Rendimiento del camote variedad Huambachero y Paramonguino.

\begin{tabular}{ccccc}
\hline Tratamiento & $\begin{array}{c}\text { Huambachero } \\
\left(\mathbf{t}^{*} \mathbf{h a}^{-\mathbf{1}} \mathbf{)}\right.\end{array}$ & $\boldsymbol{\%}$ & $\begin{array}{c}\text { Paramonguino } \\
\left(\mathbf{t}^{*} \mathbf{h a}^{-\mathbf{1}}\right)\end{array}$ & $\boldsymbol{\%}$ \\
\hline Control & 42,1 & 100,00 & 39,18 & 100,00 \\
SPR 23 días & 34,7 & 82,44 & 30,00 & 76,58 \\
SPR 33 días & 32,7 & 77,50 & 31,37 & 80,08 \\
SPR 45 días & 35,1 & 83,34 & 32,62 & 83,25 \\
\hline
\end{tabular}

Los resultados indican, que la disminución de rendimiento del cultivo en ambas variedades, regada con secado parcial de raíces (SPR) no fue muy significativa, en comparación al cultivo en ambas variedades con aplicación de riego normal (testigo), a pesar de que hubo tres inicios diferentes de riego parcial; esto nos indica que esta forma de aplicación de riego ayudaría en el ahorro de agua. La variación de rendimiento en cuanto a los tratamientos aplicados en el riego parcial, establece una tendencia de una relación directa del consumo de agua y el rendimiento (Figura 6).

\section{Consumo de agua de riego}

Para determinar la cantidad de agua se consideró un área de $0,24 \mathrm{~m}^{2}$ por gotero, lo que equivale $3,6 \times 10^{-}$ ${ }^{4}$ ha (Tabla 6).

Tabla 4. Resultados de la prueba de Tukey Huambachero.

\begin{tabular}{cccccc}
\hline Tratam. & Media & N & Tukey & ANVA & CV \\
\hline Testigo & 42,136 & 5 & A & NS & 24,0 \\
& & & & & $8 \%$ \\
SPR 23 & 34,738 & 5 & A & NS & \\
SPR 33 & 32,656 & 5 & A & NS & \\
SPR 45 & 35,115 & 5 & A & NS & \\
\hline
\end{tabular}


Tabla 5. Resultados de la prueba de Tukey Paramonguino.

\begin{tabular}{lccccc}
\hline \multicolumn{1}{c}{ Tratam. } & Media & N & Tukey & ANV & CV \\
\hline Testigo & 39,179 & 5 & A & NS & $19,05 \%$ \\
SPR 23 & 30,004 & 5 & A & NS & \\
SPR 33 & 31,373 & 5 & A & NS & \\
SPR 45 & 32,618 & 5 & A & NS & \\
\hline
\end{tabular}

En la Figura 5 el tratamiento control, se aplicó 9,66 $\mathrm{m}^{3}$ agua, equivalente a $536,89 \mathrm{~mm}\left(5368,9 \mathrm{~m}^{3} \cdot \mathrm{ha}^{-1}\right)$ y representa el $100 \%$ de necesidad hídrica. En el tratamiento del inicio del riego del secado parcial de raíces a los 23 días, se brindó $5,73 \mathrm{~m}^{3}$ de agua, equivalente a $318,55 \mathrm{~mm}\left(3185,5 \mathrm{~m}^{3} \mathrm{ha}^{-1}\right)$ que representa el 59,33\% de necesidad hídrica, ahorrando 40,67\% de agua respecto al testigo. En el tratamiento del inicio del riego del secado parcial de raíces a los 33 días, se empleó $6,17 \mathrm{~m}^{3}$ de agua que equivale a $342,95 \mathrm{~mm}(3429,5) \mathrm{m}^{3}$. ha ${ }^{-1} \mathrm{y}$ representa el $63,88 \%$ de necesidad hídrica, ahorrando $36,12 \%$ de agua respecto al testigo. Respecto al tratamiento del inicio del riego del secado parcial de raíces a los 45 días, se obtuvo un rendimiento de $35,1 \mathrm{t}$. ha ${ }^{-1}$ con un consumo de agua de $6,68 \mathrm{~m}^{3}$ lo que equivale a $371,33 \mathrm{~mm}\left(3713,3 \mathrm{~m}^{3} \mathrm{ha}^{-1}\right)$ y representa el $69,16 \%$ de necesidad hídrica, ahorrando $30,84 \%$ de agua respecto al testigo. Önder et al. (2015), en su trabajo de investigación en Turquía con el sistema de riego por goteo utilizó un consumo de agua que varió de $365 \mathrm{~mm}$ a $714 \mathrm{~mm}$. En las Figuras 6 y 7, se muestran la comparación de consumo de agua y rendimiento para ambas variedades de camote.

Se consideró el consumo de agua del testigo 5368,9 $\mathrm{m}^{3}$ ha $^{-1}$ como el $100 \%$, para la respectiva comparación con los demás tratamientos (Figura 5).

Tabla 6. Eficiencia de uso de agua en el cultivo del camote.

\begin{tabular}{|c|c|c|c|c|c|}
\hline \multirow[t]{2}{*}{ Tratamiento } & \multicolumn{2}{|c|}{ Rendimiento kg*ha-1 } & \multirow{2}{*}{ 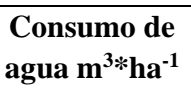 } & \multicolumn{2}{|c|}{ Eficiencia de uso del agua $\mathrm{kg}^{*} \mathrm{~m}^{-3}$} \\
\hline & Huambachero & Paramonguino & & Huambachero & Paramonguino \\
\hline Testigo & 42135,79 & 39179,33 & 5368,86 & 7,85 & 7,30 \\
\hline 23 días & 34738,33 & 30004,03 & 3185,54 & 10,91 & 9,42 \\
\hline 33 días & 32656,11 & 31373,37 & 3429,47 & 9,52 & 9,15 \\
\hline 45 días & 35115,40 & 32617,78 & 3713,26 & 9,46 & 8,78 \\
\hline
\end{tabular}

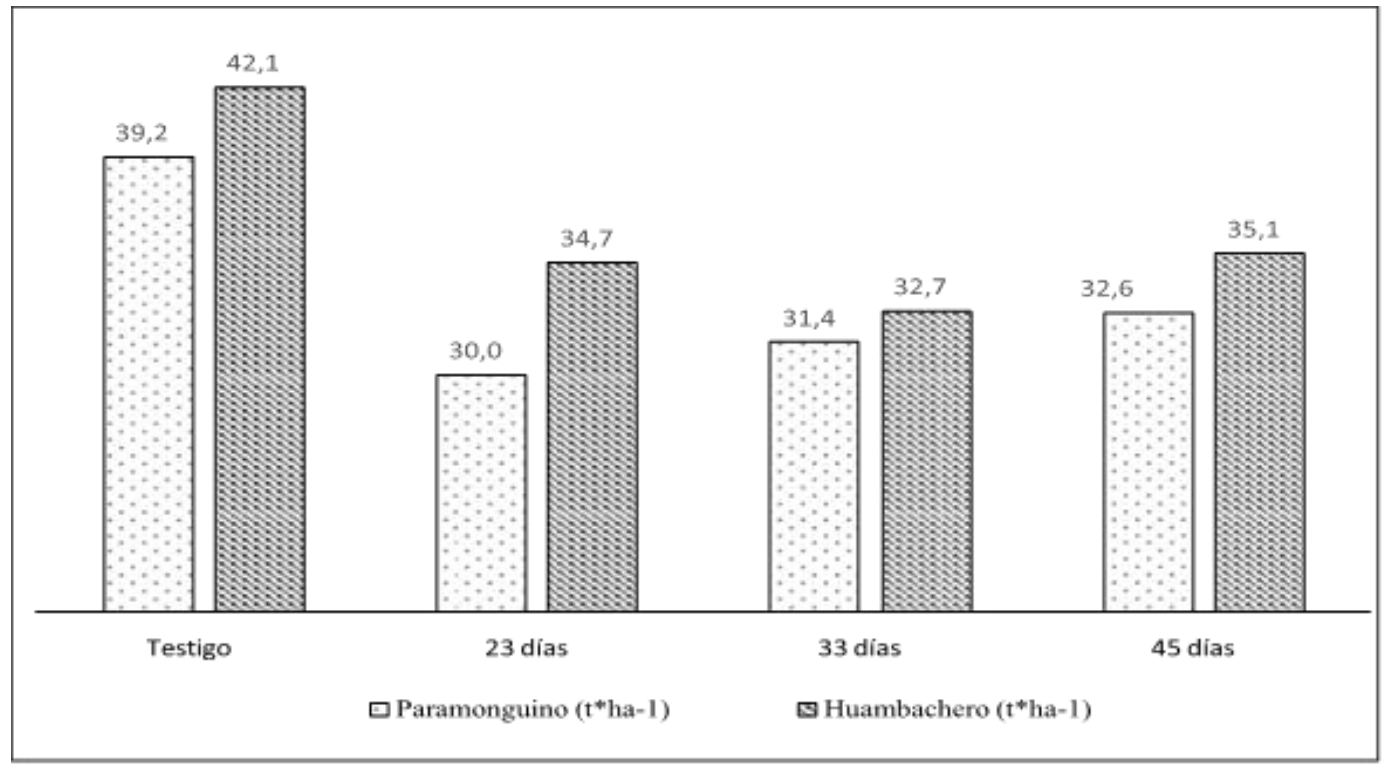

Figura 4. Comparación del rendimiento entre en las variedades de camote Huambachero y Paramonguino. 


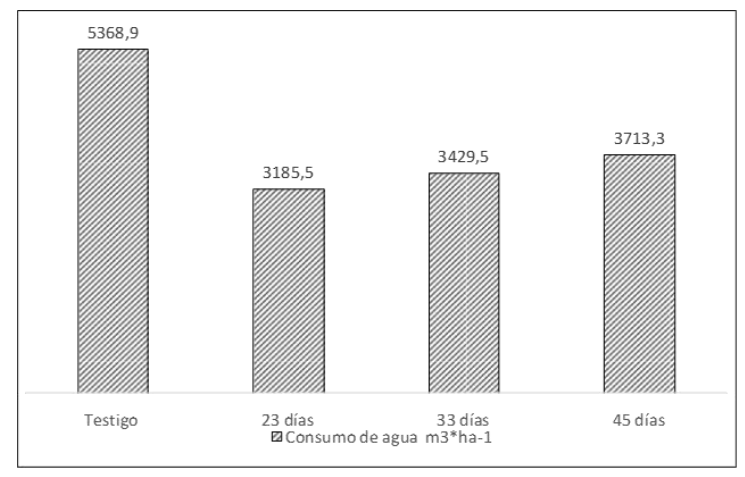

Figura 5. Aplicación de agua de riego $\mathrm{m}^{3 *} \mathrm{ha}^{-1}$

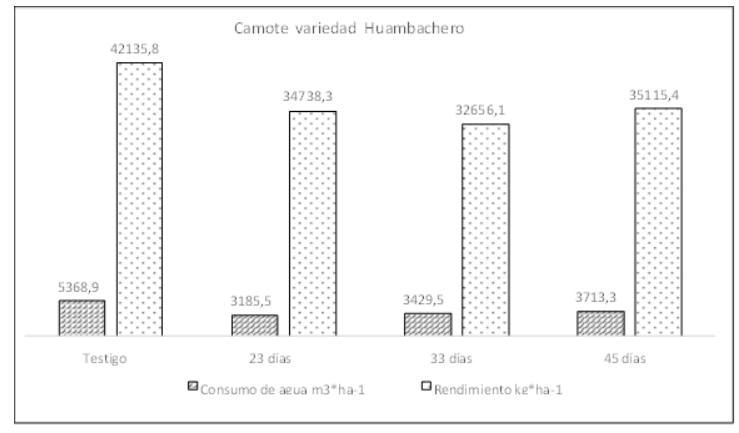

Figura 6. Comparación del rendimiento con la aplicación de agua en la variedad Huambachero.

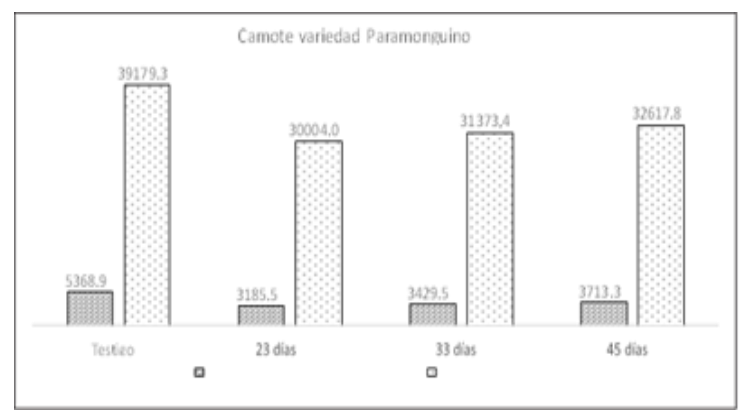

Figura 7. Comparación del rendimiento con la aplicación de agua en la variedad Paramonguino.

Los resultados indican, que el consumo de agua del cultivo para ambas variedades, fue proporcional al rendimiento del cultivo, tanto en el tratamiento control como en los tratamientos de secado parcial de raíces (SPR) de 23 días, 33 días y 45 días.

\section{Eficiencia de uso del agua por el cultivo de camote}

Los valores de la eficiencia de uso de agua se muestran en la Tabla 7. Hay una diferencia, entre los valores de la eficiencia de uso del agua entre los 4 tratamientos, tanto en variedad Huambachero como en la variedad Paramonguino, de los cuales, los valores más altos corresponden al tratamiento de 23 días, con valores de $10,91 \mathrm{~kg} \mathrm{~m}^{-3}$ y $9,42 \mathrm{~kg} \mathrm{~m}^{-3}$, para Huambachero y Paramonguino respectivamente, esto debido al menor consumo de agua en comparación con los otros tratamientos. Los valores más bajos, le corresponden al tratamiento testigo o control con 7,85 $\mathrm{kg} \mathrm{m}^{-3}$ y 7,30 $\mathrm{kg} \mathrm{m}^{-3}$, igualmente para Huambachero y Paramonguino.

Masango (2014), reporó valores de eficiencia de uso del agua que oscilaba entre $65 \mathrm{~kg} \mathrm{ha}^{-1} \mathrm{~mm}^{-1}(6,5 \mathrm{~kg}$ $\left.\mathrm{m}^{-3}\right)$ y $98 \mathrm{~kg} \mathrm{ha}^{-1} \mathrm{~mm}^{-1}\left(9,8 \mathrm{~kg} \mathrm{~m}^{-3}\right)$. Por otro lado, Bok (1998), encontró valores que estaban entre $69,83 \mathrm{~kg} \mathrm{ha}^{-1} \mathrm{~mm}^{-1}\left(6,983 \mathrm{~kg} \mathrm{~m}^{-3}\right)$ y $131,78 \mathrm{~kg} \mathrm{ha}^{-1}$ $\mathrm{mm}^{-1}\left(13,178 \mathrm{~kg} \mathrm{~m} \mathrm{~m}^{-3}\right)$, cuando realizó un experimento en Pretoria (provincia de Gauteng de Sudáfrica) sobre la respuesta del camote a diferentes regímenes de agua del suelo. En otro experimento Laurie et al. (2012) reportaron valores entre $54 \mathrm{~kg}$ $\mathrm{ha}^{-1} \mathrm{~mm}^{-1}\left(5,4 \mathrm{~kg} \mathrm{~m}^{-3}\right)$ y $70,5 \mathrm{~kg} \mathrm{ha}^{-1} \mathrm{~mm}^{-1}(7,05 \mathrm{~kg}$ $\mathrm{m}^{-3}$ ) para la variedad Resisto. Sánchez y Rivera (2018), reportaron para una lámina del 100\% en el riego por goteo convencional e intermitentes de 17,48 y $19,28 \mathrm{~kg} \mathrm{~m}^{-3}$ respetivamente en el cultivo de camote INIA 320. Por lo tanto, los valores obtenidos en la presente investigación, para el camote en las dos variedades, están dentro del rango de valores reportados por otros investigadores.

\section{CONCLUSIONES}

De acuerdo a los resultados se concluye la conveniencia del uso del SPR siendo el momento más adecuado del inicio del SPR a los 23 días después de la siembra con el fin de ahorrar 40,67\% de agua. Se recomienda realizar estudios con otras variedades de camote. 


\section{Conflictos de intereses}

Los autores firmantes del presente trabajo de investigación declaran no tener ningún potencial conflicto de interés personal o económico con otras personas $\mathrm{u}$ organizaciones que puedan influir indebidamente con el presente manuscrito.

\section{Contribuciones de los autores}

Preparación y ejecución: MS, JM, DJ, GR, JA; Desarrollo de la metodología: MS, JM, DJ, GR, JA; Concepción y diseño: MS, JM, DJ, GR, JA; Edición del artículo: MS, JM, DJ, GR, JA; Supervisión del estudio: MS, JM, DJ, GR, JA

\section{LITERATURA CITADA}

- Atwell, B.J., Kriedmann, P.E., Turnbull, C. (1999). Plants in action: adaptation in natur, performance in cultivation. Pp664. MacmillanEducationAutralia, Melboume, Australia.

- Bok, I. (1998). Response ofsweetpotto to different soil moisture regimes, MSc. (Agric). Univ. of Pretoria. Pretoria.

- Dalla, L., \& Giovanardi, R. (2000). Nitrogen use efficiency in tomato and potato as affected by wáter regime and $\mathrm{N}$ fertilisation, p.443-447.

- De Pascale, S., Costa, L.D., Vallone, S., Barbiera, G., \& Maggio, A. (2011). Increasing Water Use Efficiency in Vegetable Crop Production; From Plant to Irrigation Systems Efficiency. Hort. Tech., 21:301-308.

- Doorenbos, J., \& Kassam, A.H. (1979). Yield response to water. FAO Irrigation and drainage paper 33. FAO. Italy.

- Fereres, E., Soriano, M.A. (2007). Deficit irrigation for reducing agricultural wáter use. J. Expt. Bot. 58:147-159.

- Gomes, F., \& Carr, M. (2001). Effects of water availa-bility and vine harvesting frequency on the productivity of sweet potato in Southern Mozambique. I. Storage Root and Vine Yields, 37:523-537.

- Kang, S., \& Zhang, J. (2004). Controlled alternate partial root-zone irrigation: its physiological consequences and impacto on water use efficiency. Journal of Experimental Botany, 55(407).

- King, N., Wise, R., \& Bond, I. (2008). Fair deals for watershed services in South Africa. International Institute for Environment and Development. Natural Resource Issue 12, London.UK.

- Laurie, S.M., Faber, M., Van Jaarsveld P., Laurie, R.N., Du Plooy, C.P., \& Modisane, P.C. (2012). Beta-carotene yield and productivity of orange-fleshed sweet potato (Ipomoea batatas $\mathrm{L}$. Lam) as influenced by irrigation and fertilizer application treatments. SCIHort.,142:180-184.

- Masango. (2014). Water use efficiency of orange-fleshed sweet potato (Ipomeabatatas $L$. Lam.) Mater of Science (Agriculture) in Agronomy, University of Pretoria. July 2014.

- Mbwaga, Z., Mataa, M., \& Msabaha, M. (2007). Quality and yield stability of orange-fleshed sweet potato (Ipomoea batatas) varieties grown in different agro-ecologies. 8th African Crop Science Society Conference, El-Minia, Egypt, Ref, (32):339-345.

- Molden, D. (1997). Accouting for water and productivity. SWIN Paper 1. Colombo, Sri. Lanka: International water Management Institute, Pp. 27.

- Nedunchezhiyan, M., Byju G., \& Jata, S. K. (2012). Sweet Potato Agronomy. Fruit Vegetable and ceraeal. Science and Biotechnology. Global Sciences Books.

- Önder, D., Önder, S. ;Çali ;.\& ,.MÇalişkan, S . (2015). Influence of different irrigation methods and irrigation levels on water use efficiency, Yield, and yield attributes of sweet potatoes. Article in Fresenius Environmental Bulletin, 24(10a): 3398-3403.

- Ravi, V., Naskar, S.K., Makeshkumar, T., Binoy, B., \& Prakash, B.S. (2009). Molecular Physiology of Storage Root Formation and Development in Sweet Potato. Journal of Root Crops, 35(1):1-27.

- Somasundaram, K., \& Santhosh, V.S. (2008). A simulation model for sweet potato growth World J. Agr. Sci.4241254.

- Sánchez, M., \& Rivera, L. (2018). Aplicación de dos modalidades de riego por goteo en el crecimiento y producción de camote (Ipomoea 
batatas L.,variedad INIA 320). Anales Científicos, 79(1): 144- 150.

- Sánchez, J., \& Torrecillas, A. (1995). Riego Deficitario Controlado. Ediciones Mundi Prensa, España, 50 p.

- Shock y Welch (2013). El riego por goteo: Una introducción. Técnicas para la agricultura sostenible. 